\section{Intervenções para solucionar a superlotação nos serviços de emergência hospitalar: uma revisão sistemática}

\author{
Interventions to solve overcrowding in hospital \\ emergency services: a systematic review
}

\author{
1 Escola Nacional de Saúde \\ Pública Sergio Arouca, \\ Fundação Oswaldo Cruz, Rio \\ de Janeiro, Brasil. \\ Correspondência \\ R. J. Bittencourt \\ Escola Nacional de Saúde \\ Pública Sergio Arouca, \\ Fundção Oswaldo Cruz. \\ Rua Leopoldo Bulhões 1480, \\ Rio de Janeiro, $R J$ \\ 21041-210, Brasil. \\ robertobittencourt@ensp. \\ fiocruz.br
}

\begin{abstract}
This review discusses interventions aimed at solving the problem of overcrowding in hospital emergency services (HES), characteristic of low organizational effectiveness. In free-access and restricted-access databases, the target descriptors were "overcrowding; emergency; medicine; first aid". The survey identified 66 citations of interventions, grouped in 47 related interventions. The majority of the studies used observational designs that evaluated the results before and after interventions. Of the 47 related interventions, 34 had results for organizational performance. Four were the most frequently cited: implementation of an observation unit for patients already hospitalized and awaiting diagnosis or clinical stabilization; designation of a dedicated nursing station for the patient's admission, discharge, or transfer; establishment of protocols with indicators of operational saturation and implementation of an emergency care unit. Analyzing 21 interventions that were intended to solve overcrowding in HES and with positive results for length-of-stay, 15 were related to patient stream improvement, interfering actively and positively in patient stream. Interventions that increased access barriers or that merely improved the HES structure were ineffective.
\end{abstract}

Hospital Emergency Service; Hospital Administration; Review
Roberto José Bittencourt 1

Virginia Alonso Hortale 1

\section{Introdução}

A superlotação nos Serviços de Emergência Hospitalar (SEH) é um fenômeno mundial, caracteriza-se por: todos os leitos do SEH ocupados; pacientes acamados nos corredores; tempo de espera para atendimento acima de uma hora; alta tensão na equipe assistencial; grande pressão para novos atendimentos. Indica, em última instância, baixo desempenho do sistema de saúde, como um todo e do hospital em particular, e induz à baixa qualidade assistencial 1 .

Revisão da literatura sobre os fatores que contribuíam para a superlotação 2 , concluiu que o aumento do tempo de permanência no SEH é o principal marcador da superlotação; a falta de leitos para internação a principal causa; e o atraso no diagnóstico e tratamento, a principal conseqüência, levando ao aumento da mortalidade. Outros estudos 3,4,5 realizados em SEH mostraram resultados desfavoráveis no tratamento da pneumonia, sépsis, infarto agudo do miocárdio e apendicite relacionados com o atraso no atendimento médico. Estudos sobre a superlotação no SEH em hospitais de nível terciário apontaram resultados semelhantes. Um deles ${ }^{6}$, desenvolvido em um hospital, encontrou aumento da mortalidade hospitalar nos dez dias iniciais a partir da internação. O outro, desenvolvido em três hospitais 7, encontrou relação linear entre a superlotação no SEH e o aumento da mortalidade nos segundo, sétimo e trigésimo dias de internação. 
No Brasil, entre 2002 e 2003, o Ministério da Saúde regulamentou esse tipo de atendimento com prioridade na implantação do Serviço de Atendimento Móvel às Urgências (SAMU). No entanto, ainda existem poucos estudos sobre o desempenho do subsistema de urgência e emergência, em especial, com foco na superlotação dos SEH, suas causas e as intervenções para superação desse grave problema de saúde pública.

Dentre os poucos estudos realizados, um deles 8 discute o processo de trabalho nos SEH. Outro ${ }^{9}$ aprofunda a caracterização do que é urgência com base na opinião dos trabalhadores e usuários dos $\mathrm{SEH}$.

Levantamento recente 10 identificou no Sistema Único de Saúde (SUS) a existência de 185 hospitais com perfil de urgência e emergência. Uma parte desses hospitais participou do processo de qualificação do atendimento às urgências e emergências hospitalares, implementado pelo Ministério da Saúde, denominado QualiSUS. Artigo apresentando essa proposta 11 revelou que o padrão do atendimento é a superlotação, em especial, nos grandes centros urbanos; em alguns, com demanda de mais de mil pacientes por dia.

No presente estudo, a superlotação nos SEH será considerada como expressão de um fenômeno relacionado ao desempenho das organizações hospitalares. Como modelo conceitual será adotado o de Sicotte et al. 12, com ênfase na dimensão "efetividade organizacional", entendida como um dos elementos do desempenho organizacional e definida como: "a relação entre o serviço ofertado pela organização de saúde e os resultados obtidos" 12 (p. 26).

Assim, esta revisão tem como objetivo apresentar e discutir as intervenções voltadas para solucionar o problema da superlotação dos SEH, percebendo esse fenômeno como evidência, dentre outras, de baixa efetividade organizacional.

\section{Metodologia}

Utilizou-se como base metodológica da revisão o fluxograma proposto por Pai et al. ${ }^{13}$. As palavraschave foram definidas em quatro idiomas: inglês (overcrowding; crowding; emergency; medicine; room; department), português (superlotação; emergência; medicina; pronto-socorro), francês (surchargement; surchargés; médecine; urgence) e espanhol (congestión; servicios; urgencias). Em seguida, foram pesquisadas as bases de dados eletrônicas de livre acesso e de acesso restrito e os títulos de estudos que contivessem as palavras-chave. O critério de inclusão dos títulos foi a existência do termo "superlotação". Foram encontrados 2.992 títulos e selecionados 822, não repetidos e com referência a qualquer citação sobre superlotação em serviços de saúde para o período 2000-2007 (mês de junho). O resultado é apresentado na Tabela 1. Na seleção dos resumos, o critério de inclusão foi a existência de qualquer referência a discussões, ferramentas de gestão ou intervenções relacionadas à superlotação nos SEH. Dos 822 títulos, foram selecionados 325 resumos. O critério adotado na seleção dos textos integrais foi o de realizarem estudos empíricos sobre intervenções para solucionar a superlotação nos SEH e que apresentaram metodologia, resultado e local de realização do estudo ou descrição de modelo com foco nesse objetivo. Foram selecionados 147 artigos e 49 comunicações em congressos ou seções científicas. Durante o levantamento, foi encontrado um capítulo de livro com 28 citações não repetidas nos estudos primários, optando-se por incluí-lo na revisão.

Ao final, foram selecionados o capítulo do livro, 25 artigos e 13 comunicações. O levantamento foi concluído com 66 citações de intervenções, agrupadas em 47 intervenções afins.

A decisão sobre a seleção dos títulos, resumos e artigos foi feita por dois pesquisadores de forma consensual, portanto, a referência não consensual foi excluída do estudo.

A seguir, são apresentadas as definições adotadas na sistematização dos estudos:

- Intervenção - ação nos SEH com foco em solucionar a superlotação;

- Superlotação - situação que revela a saturação do limite operacional do SEH. De acordo com Weiss et al. 14, são os seguintes os indicadores de superlotação nos SEH: (a) 100\% de ocupação dos leitos; (b) pacientes nos corredores por causa da falta de leitos disponíveis; (c) não recebimento de ambulâncias em razão da saturação operacional; (d) sala de espera para consulta médica lotada; (e) equipe do SEH encontra-se subjetivamente no limite da exaustão; (f) mais de uma hora de espera para o atendimento médico.

- Local da intervenção - definido com base no modelo conceitual e operacional do SEH 15,16,17: input, throughput e output. No input (pressão de entrada ao SEH), consideram-se os componentes que caracterizam o atendimento no SEH pelo encaminhamento dos serviços pré-hospitalares de emergência: consultas não agendadas por causa do não-acesso no nível de atenção primária ou conveniência da pessoa, atendimento à população de risco social ou suscetível às barreiras de acesso 15 e percepção, por parte do usuário, da qualidade do atendimento no SEH 16. No throughput (pressão intrínseca do $\mathrm{SEH})$, consideram-se os componentes próprios do SEH: chegada do paciente, triagem e sala de espera, diagnóstico e tratamento no SEH, pa- 
Títulos encontrados e selecionados nas bases de dados eletrônicas de livre acesso e de acesso restrito, 2007.

\begin{tabular}{|c|c|c|}
\hline Bases de dados & Encontrados & Selecionados \\
\hline \multicolumn{3}{|l|}{ Livre acesso } \\
\hline LILACS & 2 & 0 \\
\hline MEDLINE & 252 & 119 \\
\hline Medscape & 1.068 & 230 \\
\hline Google Scholar & 704 & 279 \\
\hline Banco de Teses (CAPES) & 4 & 2 \\
\hline Cochrane & 159 & 8 \\
\hline SciELO & 103 & 5 \\
\hline Subtotal & 2.292 & 643 \\
\hline \multicolumn{3}{|l|}{ Acesso restrito (CAPES) } \\
\hline Science Direct & 81 & 48 \\
\hline Thomson Gale & 47 & 19 \\
\hline OVOID & 107 & 59 \\
\hline Emerald & 31 & 2 \\
\hline Oxford Journal & 274 & 17 \\
\hline Current & 10 & 3 \\
\hline ProQuest & 30 & 17 \\
\hline Wilson Web & 5 & 1 \\
\hline Blackwell & 115 & 13 \\
\hline Subtotal & 700 & 179 \\
\hline Total & 2.992 & 822 \\
\hline
\end{tabular}

ciente aguardando internação 15 , atendimento especializado disponível, equipe adequada de plantão, sistemas de informação disponíveis 16 . No output (pressão de saída do SEH), consideram-se os componentes que interferem na saída do paciente do SEH, como por exemplo, acesso ao nível primário de atenção, acesso a hospitais de referência ou internação domiciliar, acesso às unidades hospitalares (CTI, centro cirúrgico, leitos hospitalares) 15 , acesso aos serviços de saúde mental, centros de especialidades, acesso aos serviços de transporte 16;

- Características dos serviços - distribuídos em hospital universitário, hospital urbano, hospital comunitário, rede hospitalar e rede assistencial;

- Nível de atendimento ao trauma (Comitê de Trauma do Colégio Americano de Cirurgiões) - classificado em: nível 1 (referência regional; liderança em ensino, pesquisa e planejamento no atendimento ao trauma; tem disponíveis equipes de cirurgiões de trauma, anestesiologistas, médicos especialistas, enfermeiras e equipamentos de ressuscitação). O desempenho mínimo requerido é de 1.200 admissões/ano, atendimento de 240 pessoas vítimas de grandes traumas/ano; atendimento de 35 pessoas com grandes traumas/cirurgião. Nível 2 (mesmos critérios do nível 1, porém não se exige volume de atendimento mínimo nem ser líder em educação e pesquisa no atendimento ao trauma). Geralmente são centros de suporte no nível 1 em áreas com baixa densidade populacional. Nível 3 (prontoatendimento, avaliação, ressuscitação, cirurgia de emergência e estabilização, com transferência aos centros níveis 1 ou 2 quando necessário). Atende a populações sem acesso imediato nos níveis 1 e 2. Nível 4 (suporte avançado de vida). O objetivo principal é ressuscitar e estabilizar o paciente e providenciar sua transferência imediata para os níveis 1 ou 2;

- Características da intervenção - na estrutura, no processo e no resultado 18 ;

- As evidências para a revisão foram assim classificadas - (A) estudos randomizados; (B1) estudos observacionais antes e depois da intervenção com tratamento estatístico; (B2) estudos observacionais antes e depois da intervenção sem tratamento estatístico; (C) relato de caso; descrição de modelo; consenso de especialistas ou opinião de especialista. Cabe destacar que as intervenções do tipo C, embora não tenham resultados referidos ao desempenho organizacional, compuseram a revisão, já que auxiliaram no entendimento do modelo assistencial ao explici- 
tar a base conceitual e teórica das intervenções nos SEH.

\section{Resultados}

Das 47 intervenções afins, 34 apresentaram resultados no desempenho organizacional. Dessas, quatro tiveram maior número de citações: implantação da unidade de observação dos pacientes internados e aqueles aguardando diagnóstico ou estabilização clínica (oito citações); implantação do serviço de enfermagem dedicado à admissão, alta e transferência do paciente ou aumento da equipe de enfermagem ou implantação do núcleo de enfermagem (oito citações); instituição de protocolos com indicadores de saturação operacional do SEH (sete citações) e implantação da unidade de pronto-atendimento (cinco citações). As demais intervenções tiveram de uma a três citações. As Tabelas 2, 3 e 4 sintetizam esses achados.

Em relação ao local das intervenções, quatro resultados estão relacionados à pressão de entrada 16,19,20,21; 23 estão relacionados às pressões intrínsecas ao SEH 1,16,20,22,23,24,25,26,27,28,29,30,31,32, $33,34,35,36,37,38,39,40,41,42,43,44,45,46,47$; e sete relacionados às pressões de saída 1,16,35,48,49. As quatro intervenções mais citadas estão relacionadas às pressões intrínsecas ao SEH.

Em relação ao desenho do estudo, a "implantação do serviço de enfermagem dedicado à admissão, alta e transferência do paciente no SEH" foi a que apresentou três estudos do tipo B1 (dois com resultado negativo e um com resultado positivo). A intervenção "implantação da unidade de observação no SEH dos pacientes internados e pacientes aguardando diagnóstico ou estabilização clínica" apresentou três estudos B1; a intervenção "instituição de protocolos com indicadores de saturação operacional do SEH" apresentou dois estudos B1, ambos com resultados positivos. A intervenção "implantação da unidade de pronto-atendimento" apresentou um estudo do tipo B1. Não foram encontrados estudos randomizados tipo A.

Quanto ao porte do hospital ou da rede onde foram realizados os estudos, há equilíbrio entre os hospitais estudados. Quanto à efetividade organizacional no SEH, os dois resultados mais freqüentes foram o "tempo de permanência do paciente" e o "número de horas em desvio de ambulância”. O primeiro resultado é mais importante para a realidade brasileira, já que o segundo não é adotado como rotina.

De todas as intervenções, 21 se referiam ao “tempo de permanência no SEH”. Três delas com resultado positivo: implantação da unidade de pronto-atendimento; implantação da unidade de observação; instituição de protocolos de saturação operacional. A intervenção "expansão do número de leitos no SEH” apresentou resultado negativo, vale dizer, aumentou o tempo de permanência do paciente; por outro lado, as intervenções “implantação do serviço de enfermagem dedicado à admissão, alta e transferências do paciente no SEH" e "aumento dos leitos da unidade de pronto-atendimento" apresentaram estudos com resultados positivos e negativos. Seis intervenções estavam relacionadas às pressões de saída do SEH, e 15 às pressões intrínsecas do SEH.

\section{Discussão}

O desempenho dos serviços de saúde está sob permanente crítica e questionamento; em especial, os serviços de urgência e emergência. Não é comum entre gestores da área da saúde a utilização de revisões sistemáticas para a tomada de decisões baseada em evidências visando aperfeiçoar as intervenções, porém vem crescendo em alguns países. Em estudo realizado em Ontário, Canadá 50, a taxa de aproveitamento dos resultados de revisões sistemáticas entre gestores é de $63,1 \%$, após três meses da sua disseminação.

Qual seria o melhor desenho para estudos primários em intervenções em saúde? Há autores 51 que defendem a necessidade das evidências irem além dos ensaios clínicos randomizados, freqüentemente, pouco práticos. Propõem desenhos de estudos não randomizados baseados na plausibilidade e adequação.

Nesse sentido, já existem trabalhos que qualificam os estudos não randomizados de intervenções em saúde pela proposta denominada TREND (Transparent Reporting of Evaluations with Nonrandomized Designs) ${ }^{52}$. Neles é proposto avaliar a intervenção valendo-se de três dimensões principais: o marco teórico; a descrição das condições e o local onde ela ocorreu de forma compartilada; e o desenho da pesquisa. Por ser mais potente, o estudo baseado em cluster randomised trial 53 é sugerido para avaliar estratégias que visem melhorar a qualidade das intervenções.

Quando a randomização não for possível, são propostos estudos quase experimentais, estudos controlados antes e depois da intervenção e estudos seriados no tempo. A síntese qualitativa é indicada quando a metanálise não for adequada para sistematizar a análise dos dados. Neste caso, devem-se evitar dois tipos de erro: (a) analisar múltiplos resultados - são os revisores que definem o resultado mais importante; 
Síntese das intervenções e resultados sobre a pressão de entrada nos Serviços de Emergência Hospitalar (SEH).

\begin{tabular}{|c|c|c|c|c|c|}
\hline Intervenção & $\begin{array}{l}\text { Tipo de } \\
\text { estudo }\end{array}$ & $\begin{array}{c}\text { Local da } \\
\text { intervenção }\end{array}$ & $\begin{array}{l}\text { Característica } \\
\text { do serviço }\end{array}$ & $\begin{array}{l}\text { Características } \\
\text { da intervenção }\end{array}$ & Resultado \\
\hline $\begin{array}{l}\text { Estabelecimento de rede de } \\
\text { cooperação entre hospitais } \\
\text { em situação de desvio } \\
\text { de ambulância } 16\end{array}$ & B2 & Input & Rede hospitalar & Processo & $\begin{array}{l}\text { Diminuição do } \\
\text { tempo de desvio de } \\
\text { ambulância }\end{array}$ \\
\hline $\begin{array}{l}\text { Implantação da regulação médica } \\
\text { no atendimento pré-hospitalar } 19\end{array}$ & B2 19 & Input & Hospital universitário 19 & Estrutura & $\begin{array}{l}\text { Diminuição de } \\
\text { horas de desvio de } \\
\text { ambulância }\end{array}$ \\
\hline $\begin{array}{l}\text { Implantação de central de } \\
\text { liberação com monitoração do } \\
\text { status operacional dos SEH em } \\
\text { tempo real } 20\end{array}$ & B1 20 & & Rede hospitalar 20 & Estrutura & $\begin{array}{l}\text { Diminuição de } \\
\text { horas de desvio de } \\
\text { ambulância }\end{array}$ \\
\hline $\begin{array}{l}\text { Monitoração em tempo real via } \\
\text { Internet do status da capacidade } \\
\text { operacional dos SEH } 20\end{array}$ & B1 & Input & Rede hospitalar & Processo & $\begin{array}{l}\text { Diminuição do } \\
\text { tempo de desvio de } \\
\text { ambulância }\end{array}$ \\
\hline $\begin{array}{l}\text { Reestruturação da rede de } \\
\text { atenção: diminuição de } \\
\text { hospitais e aumento da } \\
\text { rede ambulatorial } 21\end{array}$ & B2 & Input & Rede assistencial & Estrutura & $\begin{array}{l}\text { Aumento moderado a } \\
\text { severo da superlotação } \\
\text { dos SEH (negativo) }\end{array}$ \\
\hline $\begin{array}{l}\text { Monitoração em tempo real via } \\
\text { Internet do status da capacidade } \\
\text { operacional dos SEH } 20\end{array}$ & B1 & Input & Rede hospitalar & Processo & $\begin{array}{c}\text { Diminuição do } \\
\text { tempo de desvio de } \\
\text { ambulância }\end{array}$ \\
\hline $\begin{array}{l}\text { Identificação dos pacientes } \\
\text { que procuram o SEH com mais } \\
\text { freqüência e encaminhá-los à } \\
\text { rede básica } 60,61\end{array}$ & C & Input & $\begin{array}{c}\text { Rede hospitalar 60; } \\
\text { hospital universitário } 61\end{array}$ & Processo & * \\
\hline
\end{tabular}

* Embora os estudos de tipo C não tenham apresentado resultados, foram incluídos na Tabela, pois auxiliaram no entendimento do modelo assistencial e na explicação da base conceitual e teórica da superlotação nos SEH, enquanto subsistema do sistema de saúde.

(b) confundir os dados gerados pelas unidades estudadas com os dados obtidos pelas pessoas atendidas nessas unidades. Deve-se ter em conta o efeito relatado ao invés da significância estatística (valor de p).

Foster 54 considera que os poucos estudos sobre intervenções objetivando reduzir a superlotação são inconsistentes, pois são observacionais, não experimentais e utilizam desenhos de análise simples como antes e depois da intervenção, passíveis de vieses tipo "tendência secular". E são assim categorizados: (a) aumentar recursos (aumentar o número de médicos no plantão ou o número de leitos no SEH); (b) atender fora dos SEH pessoas com agravos de baixo risco (implantar unidade de pronto-atendimento, anexo ao SEH ou serviço de avaliação no dia seguinte ao atendimento no SEH; (c) introduzir novas tecnologias (novos pontos de coleta de exames de sangue no SEH). O autor sugere que os estudos adotem as análises temporais em séries maiores ou estudos randomizados e que avaliem os resultados clínicos em vez de tempo médio de permanência.

A análise realizada por esse mesmo autor é compatível ao obtido na presente revisão. A maioria dos trabalhos foi de estudos observacionais que avaliam os resultados das intervenções 
Tabela 3

Síntese das intervenções e resultados sobre as pressões intrínsecas aos Serviços de Emergência Hospitalar (SEH).

\begin{tabular}{|c|c|c|c|c|c|}
\hline Intervenção & $\begin{array}{l}\text { Tipo de } \\
\text { estudo }\end{array}$ & $\begin{array}{l}\text { Local (dentro } \\
\text { do SEH) }\end{array}$ & $\begin{array}{l}\text { Característica } \\
\text { do serviço }\end{array}$ & $\begin{array}{l}\text { Características } \\
\text { da intervenção }\end{array}$ & Resultado \\
\hline $\begin{array}{l}\text { Expansão do número de leitos } \\
\text { no SEH } 22\end{array}$ & B2 & Throughput & $\begin{array}{l}\text { Hospital universitário } \\
\text { com nível } 1 \text { de } \\
\text { assistência ao trauma }\end{array}$ & Estrutura & $\begin{array}{c}\text { Sem alteração das } \\
\text { horas de desvio } \\
\text { de ambulância; } \\
\text { aumento do tempo de } \\
\text { permanência no SEH; } \\
\text { aumento do tempo de } \\
\text { espera para internação; } \\
\text { sem alteração no } \\
\text { número de pessoas } \\
\text { que deixaram o SEH } \\
\text { sem serem atendidos }\end{array}$ \\
\hline $\begin{array}{l}\text { Implantação da unidade } \\
\text { de pronto-atendimento } \\
16,23,24,25,26,60,62,63\end{array}$ & $\begin{array}{c}\text { B2 } 16,23,24,25 \\
\text { B1 } 26 \\
\text { C } 60,62,63\end{array}$ & Throughput & $\begin{array}{l}\text { Rede hospitalar } 16,24 \text {; } \\
\text { hospital universitário } \\
\text { nível } 2 \text { de assistência } \\
\text { ao trauma } 23 \text {; hospital } \\
\text { universitário nível } 1 \\
\text { de assistência ao } \\
\text { trauma } 25 \text {; hospital } \\
\text { universitário 26; rede } \\
\text { hospitalar } 60 ; \text { hospital } \\
\text { comunitário } 62,63\end{array}$ & Estrutura & $\begin{array}{c}\text { Redução do tempo } \\
\text { de permanência } \\
\text { no SEH 16,23,24,25; } \\
\text { satisfação das pessoas } \\
\text { foi maior 25; retorno ao } \\
\text { SEH em } 72 \text { horas foi } \\
\text { menor 25; diminuição } \\
\text { do número de pessoas } \\
\text { que deixaram o } \\
\text { SEH sem serem } \\
\text { atendidos } 25,26\end{array}$ \\
\hline $\begin{array}{l}\text { Instituição da corrida de leitos } \\
\text { no SEH e requisição de leitos } \\
\text { hospitalares pelo SEH 16,24,62 }\end{array}$ & $\begin{array}{c}\text { B2 } 16,24 \\
\text { C } 62\end{array}$ & Throughput & $\begin{array}{l}\text { Rede hospitalar 16,24; } \\
\text { hospital comunitário } 62\end{array}$ & Processo & $\begin{array}{c}\text { Diminuição da } \\
\text { instituição do } \\
\text { Código Vermelho } \\
\text { para superlotação; } \\
\text { diminuição do tempo } \\
\text { de internação do } \\
\text { paciente } 16,24\end{array}$ \\
\hline $\begin{array}{l}\text { Implantação da unidade de } \\
\text { atendimento a pacientes em } \\
\text { situação crítica } 24,27,60\end{array}$ & $\begin{array}{l}\text { B2 } 24 \\
\text { B1 } 27 \\
\text { C } 60\end{array}$ & Throughput & $\begin{array}{c}\text { Rede } \\
\text { hospitalar 24; hospital } \\
\text { universitário 27; rede } \\
\text { hospitalar } 60\end{array}$ & Estrutura & $\begin{array}{l}\text { Diminuição do tempo } \\
\text { de permanência no } \\
\text { SEH 24; diminuição das } \\
\text { horas de desvio de } \\
\text { ambulância; diminuição } \\
\text { do número de pessoas } \\
\text { que deixaram o } \\
\text { SEH sem serem } \\
\text { atendidos } 27\end{array}$ \\
\hline
\end{tabular}

(continua) 


\begin{tabular}{|c|c|c|c|c|c|}
\hline Intervenção & $\begin{array}{l}\text { Tipo de } \\
\text { estudo }\end{array}$ & $\begin{array}{c}\text { Local (dentro } \\
\text { do SEH) }\end{array}$ & $\begin{array}{c}\text { Característica } \\
\text { do serviço }\end{array}$ & $\begin{array}{l}\text { Características } \\
\text { da intervenção }\end{array}$ & Resultado \\
\hline $\begin{array}{l}\text { Implantação da unidade de } \\
\text { observação no SEH } \\
\text { dos pacientes internados e } \\
\text { pacientes aguardando definição } \\
\text { diagnóstica ou estabilização } \\
\text { clínica } 1,24,28,29,30,31,32,33,62,63\end{array}$ & $\begin{array}{c}\text { B2 } 1,24,28, \\
29,30,32 \\
\text { B1 } 31,32,33 \\
\text { C } 62,63\end{array}$ & 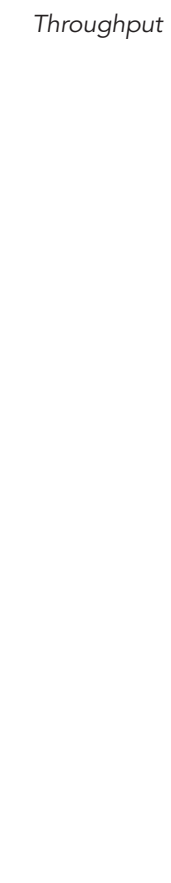 & $\begin{array}{l}\text { Rede hospitalar 1,24; } \\
\text { hospital comunitário } \\
28,62 \text {; hospital } \\
\text { universitário 29,63; rede } \\
\text { hospitalar 1,33; hospital } \\
\text { universitário 30,31,32 }\end{array}$ & Estrutura & $\begin{array}{l}\text { Diminuição do tempo } \\
\text { de permanência } \\
\text { do paciente no } \\
\text { SEH 24; melhoria do } \\
\text { desempenho clínico; } \\
\text { diminuição da carga } \\
\text { de trabalho; redução } \\
\text { no tempo de início de } \\
\text { manejo do paciente } 28 ; \\
\text { diminuição da carga de } \\
\text { trabalho; sem alteração } \\
\text { na satisfação do } \\
\text { usuário } 29 ; \text { diminuição } \\
\text { do tempo de desvio } \\
\text { de ambulância } 1,33 ; \\
\text { redução do número } \\
\text { de internações } 30 ; \text { sem } \\
\text { redução do tempo } \\
\text { de permanência } \\
\text { hospitalar } 31 ; \text { aumento } \\
\text { da oferta de leitos } \\
\text { internos } 32\end{array}$ \\
\hline $\begin{array}{l}\text { Implantação da gerência de fluxo } \\
\text { de pacientes do SEH para } \\
\text { os leitos de internação } \\
\text { (bed czar) } 16,34\end{array}$ & B2 & Throughput & $\begin{array}{l}\text { Rede hospitalar } 16 \\
\text { hospital urbano } 34\end{array}$ & Estrutura & $\begin{array}{l}\text { Diminuição do tempo } \\
\text { de espera para } \\
\text { avaliação médica do } \\
\text { paciente internado; } \\
\text { diminuição do tempo } \\
\text { de permanência do } \\
\text { paciente no SEH; } \\
\text { diminuição do tempo } \\
\text { para internação do } \\
\text { paciente } 16,34\end{array}$ \\
\hline
\end{tabular}

(continua)

antes e depois. Para estudos de intervenção em organizações de saúde, esse dado, por si só, já mostra que o padrão-ouro pode não ser o mesmo adotado em ensaios clínicos.

A opção desta revisão foi valorizar os dados encontrados, sistematizando as intervenções mais consistentes e realizar uma síntese qualitativa, pois, como afirmou Bastos 55 (p. 1252): “Enfim, há lugar ao sol para as diferentes vertentes de revisão de achados e conceitos. Que venham novas revisões, revisões sistemáticas e ensaios, desde que pautados na qualidade e legibilidade. Serão, os três, muito bem vindos!".

Esta revisão baseou-se na análise das intervenções para solucionar a superlotação nos SEH e que tiveram resultados favoráveis no evento principal - tempo de permanência no SEH (total de 21); seis não têm relação com a melhoria do fluxo, seja no próprio SEH ou nos setores do hospital. As demais, 15 intervenções, interferem ativa e positivamente no fluxo dos pacientes. Destacam-se: unidade de pronto-atendimento; "bed czar" ou gerente de leitos; coordenação de altas do SEH; prontuários eletrônicos; equipes de alto desempenho clínico; laboratório-satélite; indicadores de saturação operacional; censo de vagas e demandas; encaminhamento rápido para sala de observação clínica; busca ativa dos pacientes internados no SEH; coordenação de alta hospitalar; pré-alta; sala de alta; cancelamento de internações eletivas; redistribuição de cirurgias eletivas. 
Tabela 3 (continuação)

\begin{tabular}{|c|c|c|c|c|c|}
\hline Intervenção & $\begin{array}{l}\text { Tipo de } \\
\text { estudo }\end{array}$ & $\begin{array}{l}\text { Local (dentro } \\
\text { do SEH) }\end{array}$ & $\begin{array}{l}\text { Característica } \\
\text { do serviço }\end{array}$ & $\begin{array}{l}\text { Características } \\
\text { da intervenção }\end{array}$ & Resultado \\
\hline $\begin{array}{l}\text { Implantação do serviço de } \\
\text { enfermagem dedicado à } \\
\text { admissão, alta e transferências } \\
\text { do paciente no SEH 1,16,24; } \\
\text { ou aumento da equipe de } \\
\text { enfermagem no SEH } 26,35,36,37 \text {; } \\
\text { ou implantação do núcleo de } \\
\text { enfermagem do SEH } 38\end{array}$ & $\begin{array}{l}\text { B2 } 1,16,24,35,38 \\
\text { B1 } 26,35,37\end{array}$ & Throughput & $\begin{array}{c}\text { Rede hospitalar 16,24,37; } \\
\text { hospital universitário } \\
\text { 26,36; hospital } \\
\text { urbano 1,38; hospital } \\
\text { comunitário } 35\end{array}$ & Estrutura & $\begin{array}{l}\text { Diminuição do tempo } \\
\text { para internação } \\
\text { do paciente 16; } \\
\text { diminuição do tempo } \\
\text { de avaliação médica } \\
\text { do paciente internado; } \\
\text { diminuição do tempo } \\
\text { de permanência do } \\
\text { paciente no SEH } \\
\text { 1,24,37,38; relação } \\
\text { negativa com os } \\
\text { pacientes que } \\
\text { deixaram o SEH sem } \\
\text { serem avaliados pelo } \\
\text { médico } 26 ; \text { diminuição } \\
\text { do tempo de } \\
\text { atendimento médico } \\
\text { 26,35; aumento do } \\
\text { tempo de permanência } \\
\text { do paciente no } \\
\text { SEH 36; aumento do } \\
\text { tempo para internação } \\
36 ; \text { diminuição do } \\
\text { tempo de espera no } \\
\text { SEH } 37\end{array}$ \\
\hline $\begin{array}{l}\text { Melhoria da rotatividade do } \\
\text { atendimento nos serviços } \\
\text { laboratoriais e radiologia } 35\end{array}$ & B2 & Throughput & Hospital comunitário & Processo & $\begin{array}{l}\text { Diminuição do tempo } \\
\text { para o atendimento } \\
\text { médico }\end{array}$ \\
\hline $\begin{array}{l}\text { Implantação da coordenação das } \\
\text { altas no SEH 1,16,39 }\end{array}$ & B2 & Throughput & $\begin{array}{l}\text { Rede hospitalar 16; } \\
\text { hospital comunitário } \\
\text { nível } 2 \text { de atenção } \\
\text { ao trauma 1; rede } \\
\text { assistencial } 39\end{array}$ & Estrutura & $\begin{array}{c}\text { Diminuição do número } \\
\text { de horas em desvio } \\
\text { de ambulância 16; } \\
\text { diminuição do tempo } \\
\text { de permanência no } \\
\text { SEH 1,39 }\end{array}$ \\
\hline $\begin{array}{l}\text { Criação de incentivos financeiros } \\
\text { para médicos e enfermeiros } \\
\text { promoverem a alta hospitalar ou } \\
\text { diminuírem a superlotação } 1,16\end{array}$ & $\begin{array}{l}\text { B2 } 16 \\
\text { C } 1\end{array}$ & Throughput & $\begin{array}{l}\text { Rede hospitalar }{ }^{16} \\
\text { hospital urbano }{ }^{1}\end{array}$ & Estrutura & $\begin{array}{l}\text { Diminuição do tempo } \\
\text { de internação } 16\end{array}$ \\
\hline $\begin{array}{l}\text { Utilização de prontuário } \\
\text { eletrônico; encaminhamentos } \\
\text { intra-hospitalares; disponibilização } \\
\text { de resultados de exame; } \\
\text { ou melhoria no sistema de } \\
\text { comunicação intra-hospitalar } 40\end{array}$ & B2 & Throughput & Hospital comunitário & Estrutura & $\begin{array}{c}\text { Diminuição do tempo } \\
\text { de permanência } \\
\text { no SEH }\end{array}$ \\
\hline
\end{tabular}

(continua) 
Tabela 3 (continuação)

\begin{tabular}{|c|c|c|c|c|c|}
\hline Intervenção & $\begin{array}{l}\text { Tipo de } \\
\text { estudo }\end{array}$ & $\begin{array}{c}\text { Local (dentro } \\
\text { do SEH) }\end{array}$ & $\begin{array}{c}\text { Característica } \\
\text { do serviço }\end{array}$ & $\begin{array}{l}\text { Características } \\
\text { da intervenção }\end{array}$ & Resultado \\
\hline $\begin{array}{l}\text { Implantação de Equipe de Alto } \\
\text { Desempenho Clínico no SEH } \\
1,24,29\end{array}$ & $\begin{array}{c}\text { B2 } 24,29 \\
\text { C } 1\end{array}$ & Throughput & $\begin{array}{l}\text { Rede hospitalar 24; } \\
\text { hospital universitário 29; } \\
\text { hospital comunitário } 1\end{array}$ & Estrutura & $\begin{array}{c}\text { Diminuição do } \\
\text { tempo para realizar } \\
\text { a internação 24; } \\
\text { diminuição da carga de } \\
\text { trabalho médico sem } \\
\text { aumento da satisfação } \\
\text { do usuário } 29\end{array}$ \\
\hline $\begin{array}{l}\text { Aumento do número de } \\
\text { leitos da unidade de } \\
\text { pronto-atendimento } 41\end{array}$ & B2 & Throughput & Hospital comunitário & Estrutura & $\begin{array}{l}\text { O tempo de } \\
\text { permanência no } \\
\text { SEH ficou inalterado, } \\
\text { apesar do aumento } \\
\text { do número de } \\
\text { atendimentos; a } \\
\text { satisfação do paciente } \\
\text { aumentou }\end{array}$ \\
\hline $\begin{array}{l}\text { Implantação de setor dedicado à } \\
\text { requisição de exames no SEH } 1 \\
\text { ou implantação de laboratório- } \\
\text { satélite no SEH } 42\end{array}$ & B2 1,42 & Throughput & $\begin{array}{c}\text { Rede hospitalar 1; } \\
\text { hospital universitário } 42\end{array}$ & Estrutura & $\begin{array}{l}\text { Diminuição do } \\
\text { tempo de espera para } \\
\text { receber resultado dos } \\
\text { exames } 1 \text {; diminuição } \\
\text { do tempo de } \\
\text { permanência no SEH; } \\
\text { sem efeito nas pessoas } \\
\text { não internadas no } \\
\text { SEH } 42\end{array}$ \\
\hline $\begin{array}{l}\text { Implantação de setor para } \\
\text { procedimentos cirúrgicos simples } \\
\text { no SEH } 1\end{array}$ & B2 & Throughput & Hospital urbano & Estrutura & $\begin{array}{c}\text { Aumento dos } \\
\text { procedimentos } \\
\text { cirúrgicos; diminuição } \\
\text { do tempo de espera } \\
\text { para as cirurgias de } \\
\text { emergência e aumento } \\
\text { da oferta de leitos }\end{array}$ \\
\hline $\begin{array}{l}\text { Capacitação das equipes da } \\
\text { unidade de pronto-atendimento } \\
\text { do SEH }{ }^{1}\end{array}$ & B2 & Throughput & Rede hospitalar & Estrutura & $\begin{array}{l}\text { Diminuição do tempo } \\
\text { de permanência do } \\
\text { paciente no SEH e } \\
\text { diminuição do tempo } \\
\text { entre a chegada do } \\
\text { paciente no SEH e sua } \\
\text { internação }\end{array}$ \\
\hline $\begin{array}{l}\text { Utilização da Teoria das } \\
\text { Filas } 35,43,63\end{array}$ & $\begin{array}{c}\text { B2 } 35,43 \\
\text { C } 63\end{array}$ & Throughput & $\begin{array}{c}\text { Hospital } \\
\text { comunitário } 35 ; \\
\text { hospital urbano } 43,63\end{array}$ & Processo & $\begin{array}{l}\text { Diminuição do tempo } \\
\text { de atendimento } \\
\text { médico } 35 \text {; diminuição } \\
\text { do número de pessoas } \\
\text { que deixaram o } \\
\text { SEH sem serem } \\
\text { atendidas } 43\end{array}$ \\
\hline
\end{tabular}

(continua) 
Tabela 3 (continuação)

\begin{tabular}{|c|c|c|c|c|c|}
\hline Intervenção & $\begin{array}{l}\text { Tipo de } \\
\text { estudo }\end{array}$ & $\begin{array}{c}\text { Local (dentro } \\
\text { do SEH) }\end{array}$ & $\begin{array}{l}\text { Característica } \\
\text { do serviço }\end{array}$ & $\begin{array}{l}\text { Características } \\
\text { da intervenção }\end{array}$ & Resultado \\
\hline $\begin{array}{l}\text { Instituição de protocolos com } \\
\text { indicadores de saturação } \\
\text { operacional do SEH } \\
1,16,20,24,39,44,45,64\end{array}$ & $\begin{array}{l}\text { B2 } 1,16,24,39,45 \\
\text { B1 } 20,44 \\
\text { C } 64\end{array}$ & Throughput & $\begin{array}{l}\text { Rede hospitalar } 16,20,24 ; \\
\text { rede assistencial 1,39; } \\
\text { hospital urbano 44,64; } \\
\text { hospital urbano } \\
\text { nível } 2 \text { de atenção } \\
\text { ao trauma } 45\end{array}$ & Processo & $\begin{array}{l}\text { Diminuição de } \\
\text { horas de desvio de } \\
\text { ambulância 16,20,24; } \\
\text { diminuição do tempo } \\
\text { para internação 1,39; } \\
\text { diminuição da } \\
\text { instalação do } \\
\text { Código Vermelho 44; } \\
\text { diminuição do tempo } \\
\text { de permanência no } \\
\text { SEH; diminuição do } \\
\text { tempo de admissão } 45\end{array}$ \\
\hline $\begin{array}{l}\text { Instituição de protocolos clínicos } \\
\text { - operacionais no SEH } 46\end{array}$ & B2 & Throughput & Rede hospitalar & Processo & $\begin{array}{c}\text { Melhoria dos } \\
\text { parâmetros clínicos; } \\
\text { diminuição do número } \\
\text { de atendimentos às } \\
\text { urgências }\end{array}$ \\
\hline $\begin{array}{l}\text { Instituição de censo de demandas } \\
\text { e ofertas de vagas } 24,60\end{array}$ & $\begin{array}{l}\text { B2 } 24 \\
\text { C } 60\end{array}$ & Throughput & Rede hospitalar 24,60 & Processo & $\begin{array}{c}\text { Diminuição do tempo } \\
\text { de permanência no } \\
\text { SEH } 24\end{array}$ \\
\hline $\begin{array}{l}\text { Encaminhamento do paciente } \\
\text { diretamente à unidade de } \\
\text { observação do SEH 1,47 }\end{array}$ & $\begin{array}{c}\text { B2 } 47 \\
\text { C } 1\end{array}$ & Throughput & $\begin{array}{l}\text { Hospital universitário } \\
\text { nível } 1 \text { de atenção ao } \\
\text { trauma } 47\end{array}$ & Processo & $\begin{array}{c}\text { Diminuição do tempo } \\
\text { de permanência no } \\
\text { SEH } 47\end{array}$ \\
\hline $\begin{array}{l}\text { Estabelecimento de ferramenta } \\
\text { de gestão baseada em mudanças } \\
\text { de ciclos rápidos } 47\end{array}$ & B2 & Throughput & Hospital urbano & Processo & $\begin{array}{l}\text { Implantação de novos } \\
\text { processos institucionais }\end{array}$ \\
\hline $\begin{array}{l}\text { Estabelecimento da ferramenta } \\
\text { seis sigma } 1\end{array}$ & B2 & Throughput & Hospital universitário & Processo & $\begin{array}{l}\text { Atraso no atendimento } \\
\text { médico e de } \\
\text { enfermagem }\end{array}$ \\
\hline $\begin{array}{l}\text { Implantação do Comitê Gestor } \\
\text { Hospitalar e dos Comitês de } \\
\text { apoio da Emergência e das } \\
\text { Unidades Internas } 1\end{array}$ & C & Throughput & Hospital urbano & Estrutura & * \\
\hline $\begin{array}{l}\text { Utilização de software de } \\
\text { simulação de fluxo no SEH } 46\end{array}$ & c & Throughput & Hospital comunitário & Estrutura & * \\
\hline $\begin{array}{l}\text { Envolvimento dos gerentes } \\
\text { hospitalares na identificação dos } \\
\text { problemas e soluções para a } \\
\text { superlotação no SEH } 1\end{array}$ & C & Throughput & & Processo & * \\
\hline
\end{tabular}

(continua) 


\begin{tabular}{|c|c|c|c|c|c|}
\hline Intervenção & $\begin{array}{l}\text { Tipo de } \\
\text { estudo }\end{array}$ & $\begin{array}{l}\text { Local (dentro } \\
\text { do SEH) }\end{array}$ & $\begin{array}{l}\text { Característica } \\
\text { do serviço }\end{array}$ & $\begin{array}{l}\text { Características } \\
\text { da intervenção }\end{array}$ & Resultado \\
\hline $\begin{array}{l}\text { Monitoramento do desempenho } \\
\text { do SEH com indicadores clínicas } \\
\text { e operacionais integrados aos } \\
\text { indicadores de desempenho do } \\
\text { hospital } 1\end{array}$ & C & Throughput & & Processo & * \\
\hline $\begin{array}{l}\text { Estabelecimento de ferramenta } \\
\text { de gestão baseada: (a) no conflito } \\
\text { de demandas; (b) na análise } \\
\text { do impacto de intervenções } \\
\text { equivocadas; (c) na análise da raiz } \\
\text { causal de eventos sentinelas; (d) } \\
\text { na adequação dos profissionais às } \\
\text { funções } 1\end{array}$ & C & Throughput & & Processo & * \\
\hline $\begin{array}{l}\text { Estabelecimento de ferramenta } \\
\text { de gestão baseada no } \\
\text { gerenciamento da cadeia de } \\
\text { suprimentos } 1\end{array}$ & C & Throughput & & Processo & * \\
\hline $\begin{array}{l}\text { Estabelecimento de ferramenta } \\
\text { de gestão baseada no controle } \\
\text { estatístico do processo de } \\
\text { trabalho e identificação de } \\
\text { "gargalos" } 54,65\end{array}$ & C & Throughput & & Processo & * \\
\hline
\end{tabular}

Bradley 2, em sua revisão, descreveu 68 intervenções encontradas em 102 citações (9 intervenções sobre a entrada do paciente no SEH; 28 sobre a eficiência do SEH e 31 sobre o acesso dos pacientes aos leitos hospitalares). As intervenções que interferiam na saída dos pacientes dos SEH representavam $46 \%$ das intervenções descritas. Portanto, confirmam os achados na revisão sistemática em tela. Possivelmente, as intervenções com foco na saída do paciente do SEH poderiam ser monitoradas por um Núcleo Interno de Regulação, como sugerida em alguns estudos 56. Não foi observada ênfase nas intervenções que aumentam as barreiras de acesso aos SEH para diminuir a superlotação, possivelmente desmistificando como efetivas medidas dessa natureza.

A adoção do modelo de intervenção, fundamentado na organização dos SEH em três ambientes (entrada, dentro e saída), encontrado nas referências citadas é, todavia, distinta do modelo de atenção às emergências baseado na classificação de risco (clínico e cirúrgico) 5 . O modelo centrado na classificação de risco 56 organiza os SEH em dois fluxos: o azul e o vermelho. Os ambientes do fluxo azul têm a finalidade de atender a uma determinada seqüência: acolhimento, classificação de risco, consulta médica, sala de procedimentos de baixa complexidade e sala de observação até 12 horas. Os ambientes do fluxo vermelho atendem a outra seqüência: área de reanimação, unidade de estabilização com cuidados intensivos e sala de observação até 24 horas. As equipes do fluxo azul têm perfil adequado ao atendimento de grande pressão de porta, organizada em plantões. No fluxo vermelho as equipes têm perfil para o atendimento intensivo e procedimentos invasivos, preferencialmente, organizada horizontalmente para aumentar o vínculo e responsabilização na conduta com os pacientes.

A distinção entre esses dois modelos permite estabelecer a complementaridade entre eles e sugerir um terceiro modelo que aumente a capacidade operacional do SEH e integre-o ao conjunto da organização hospitalar 20. Essa perspectiva, possivelmente, proporcionaria maior 
Síntese das intervenções e resultados sobre as pressões de saída no Serviço de Emergência Hospitalar (SEH).

\begin{tabular}{|c|c|c|c|c|c|}
\hline Intervenção & $\begin{array}{c}\text { Evidência } \\
\text { metodológica }\end{array}$ & $\begin{array}{l}\text { Local } \\
\text { (saída) }\end{array}$ & $\begin{array}{l}\text { Porte do } \\
\text { serviço }\end{array}$ & $\begin{array}{l}\text { Características } \\
\text { da intervenção }\end{array}$ & Resultado \\
\hline $\begin{array}{l}\text { Instituição da busca ativa do } \\
\text { paciente do SEH pelas unidades } \\
\text { de internação } 16\end{array}$ & B2 & Output & Rede hospitalar & Processo & $\begin{array}{c}\text { Diminuição do tempo } \\
\text { de permanência no } \\
\text { SEH }\end{array}$ \\
\hline $\begin{array}{l}\text { Criação do coordenador da alta } \\
\text { hospitalar } 16\end{array}$ & B2 & Output & Rede hospitalar & Estrutura & $\begin{array}{c}\text { Diminuição do tempo } \\
\text { de permanência no } \\
\text { SEH }\end{array}$ \\
\hline Instituição da pré-alta 16 & B2 & Output & Rede hospitalar & Processo & $\begin{array}{c}\text { Diminuição do tempo } \\
\text { de permanência no } \\
\text { SEH }\end{array}$ \\
\hline Criação da sala de alta 16 & B2 & Output & Rede hospitalar & Estrutura & $\begin{array}{c}\text { Diminuição do tempo } \\
\text { de permanência no } \\
\text { SEH }\end{array}$ \\
\hline $\begin{array}{l}\text { Ampliação da ofertas de leitos } \\
\text { hospitalares sem especificação } 35 \\
\text { ou através do cancelamento das } \\
\text { internações eletivas } 48\end{array}$ & $\begin{array}{l}\text { B2 } 35 \\
\text { B1 } 48\end{array}$ & Output & $\begin{array}{l}\text { Hospital comunitário } 35 \text {; } \\
\text { hospital universitário } 48\end{array}$ & Processo & $\begin{array}{l}\text { Diminuição do tempo } \\
\text { de atendimento para o } \\
\text { médico } 35 \text {; diminuição } \\
\text { da média de ocupação } \\
\text { hospitalar; diminuição }\end{array}$ \\
\hline $\begin{array}{l}\text { Redistribuição das cirurgias } \\
\text { eletivas no centro cirúrgico para } \\
\text { evitar conflito com as demandas } \\
\text { do SEH } 49\end{array}$ & B2 & Output & Hospital urbano & Processo & $\begin{array}{l}\text { da ocupação no SEH; } \\
\text { diminuição do tempo } \\
\text { de espera no SEH } 48\end{array}$ \\
\hline $\begin{array}{l}\text { Redesenho do fluxo do paciente } \\
\text { no SEH } 1\end{array}$ & B1 & Output & Hospital urbano & Processo & $\begin{array}{c}\text { Diminuição do } \\
\text { tempo em desvio } \\
\text { de ambulância e } \\
\text { diminuição do tempo } \\
\text { do paciente no SEH } \\
\text { Diminuição no tempo } \\
\text { de permanência no } \\
\text { SEH }\end{array}$ \\
\hline $\begin{array}{l}\text { Instituição de equipe de } \\
\text { internistas para acelerar a } \\
\text { disponibilidade de leitos } \\
\text { hospitalares } 62\end{array}$ & C & Output & Hospital comunitário & Estrutura & * \\
\hline $\begin{array}{l}\text { Transferência aos serviços de } \\
\text { saúde pós-alta para agilizar a alta } \\
\text { hospitalar } 64\end{array}$ & C & Output & & Processo & * \\
\hline
\end{tabular}

* Embora os estudos de tipo C não tenham apresentado resultados, foram incluídos na Tabela, pois auxiliaram no entendimento do modelo assistencial e na explicação da base conceitual e teórica da superlotação nos SEH, enquanto subsistema do sistema de saúde. 
sinergia entre as intervenções e seria mais efetiva para eliminar a superlotação.

Tanto a revisão de Bradley ${ }^{2}$ quanto a presente são convergentes em seus resultados, corroborando a seguinte afirmação de Asplin \& Magid 57 (p. 273): "hospitais que tiveram maior sucesso em diminuir a superlotação nos SEH foram aqueles que reconheceram o problema do fluxo do paciente em todo o hospital e definiram iniciativas para tirá-lo do SEH de forma mais eficiente". Significa dizer que o grande número de atendimentos nos SEH indica baixo desempenho do sistema de saúde, e a superlotação dos SEH revela o baixo desempenho do hospital e sua rede. Tratase, portanto, de dois fenômenos distintos, que podem ou não interagir. Pode-se ter o primeiro fenômeno sem o segundo, assim como o segundo sem o primeiro. Entretanto, ainda prevalece a ocorrência simultânea dos dois fenômenos nos SEH.

Para futuros estudos seria importante avaliar o resultado assistencial das intervenções identificadas nesta revisão. Barlett et al. 46 sugerem indicadores clínico-operacionais para avaliar tais resultados nos SEH. No Brasil, o Programa Nacional de Avaliação em Serviços de Saúde (PNASS2007), desenvolvido pelo Ministério da Saúde em parceria com a Agência Nacional de Vigilância Sanitária (ANVISA) 58, utiliza três indicadores hospitalares: tempo médio de permanência, taxa de ocupação e taxa de mortalidade. Com eles, poderiam ser testadas as mudanças nos processos de trabalho, nas estruturas dos SEH e particularmente avaliar a relação entre a diminuição da superlotação e a redução das mortes evitáveis nos SEH.

\section{Conclusão}

Com esta revisão pretendeu-se contribuir para inserir o tema da superlotação dos SEH na agenda das pesquisas em saúde pública no Brasil. É desproporcional a dimensão do problema para o pouco que se tem estudado. Não há teses e dissertações sobre o assunto. A solução desse problema, relacionado com a efetividade organizacional, é factível. Deve-se avançar no aprofundamento de todos os aspectos e oferecer aos envolvidos, na tomada de decisão, especialmente os gerentes dos hospitais de urgência e emergência, as melhores evidências para resolver a superlotação nos SEH.

Estudo de Lemieux-Charles \& Champagne 59 cita três aspectos relacionados ao conhecimento e à busca de evidências, necessários para compreender esta revisão. O primeiro é a necessidade de comprometer o profissional com as mudanças organizacionais, sociais e culturais, mais do que procurar um "tiro mágico" (magic bullet) para as mudanças do comportamento individual. O segundo é a necessidade de desenvolver métodos que encorajem a tomada de decisão baseada em evidências, ajustando o contexto em que o profissional atua com a inovação a ser implementada. E, por último, a necessidade de ver a tomada de decisão baseada em evidências como um processo social para além de um objetivo técnico.

\section{Resumo}

Esta revisão discute as intervenções voltadas para solucionar o problema da superlotação dos Serviços de Emergência Hospitalar (SEH), como evidência de baixa efetividade organizacional. Em bases de dados eletrônicas de livre acesso e acesso restrito, os descritores buscados foram "superlotação; emergência; medicina; pronto-socorro". O levantamento identificou 66 citações de intervenções, agrupadas em 47 intervenções afins. A maioria dos trabalhos teve como desenho os estudos observacionais que avaliaram os resultados das intervenções antes e depois. As mais citadas: implantação da unidade de observação dos pacientes graves; implantação do serviço de enfermagem dedicado à admissão, alta e transferência do paciente; instituição de protocolos de saturação operacional e implantação da unidade de pronto-atendimento. Na análise das 21 intervenções para solucionar a superlotação nos SEH, que tiveram resultados favoráveis no evento principal - tempo de permanência no SEH -, 15 tinham relação com a melhoria do fluxo no próprio SEH ou nos setores do hospital, interferindo ativa e positivamente no fluxo interno dos pacientes. As intervenções que aumentaram as barreiras de acesso aos SEH, ou que apenas melhoram a estrutura dos SEH não foram efetivas.

Serviço Hospitalar de Emergência; Administração Hospitalar; Revisão 


\section{Colaboradores}

Ambos os autores participaram igualmente das etapas de desenho, coleta de dados, análise e interpretação dos dados e da elaboração do artigo.

\section{Referências}

1. Committee on the Future of Emergency Care in the United States Health System. Hospital-based emergency care: at the breaking point. Washington DC: National Academies Press; 2007.

2. Bradley VM. Placing Emergency Department crowding on the decision agenda. J Emerg Nurs 2005; 31:247-58.

3. Graff L, Stevens C, Spaite D, Foody J. Measuring and improving quality in emergency medicine. Acad Emerg Med 2002; 9:1091-107.

4. Schull M, Vermeulen M, Slaughter G, Morrison L, Daly P. Emergency Department crowding and thrombolysis delays in acute myocardial infarction. Ann Emerg Med 2004; 44:577-85.

5. Magid DJ, Asplin BR, Wears RL. The quality gap: Searching for the consequences of emergency department crowding. Ann Emerg Med 2004; 44: 586-8.

6. Richardson DB. Increase in patient mortality at 10 days associated with emergency department overcrowding. Med J Aust 2006; 184:213-6.

7. Sprivulis PC, Da Silva JA, Jacobs IG, Frazer ARL, Jelinek GA. The association between hospital overcrowding and mortality among patients admitted via Western Australian emergency departments. Med J Aust 2006; 184:208-12.

8. Deslandes SF. Frágeis deuses: profissionais de emergência entre os danos da violência e a recriação da vida. Rio de Janeiro: Editora Fiocruz; 2002.

9. Giglio-Jacquemot A. Urgências e emergências em saúde: perspectivas de profissionais e usuários. Rio de Janeiro: Editora Fiocruz; 2005.

10. Sá DA. Proposta metodológica para classificação dos hospitais de urgência/emergência do SUS. Brasília: Secretaria Executiva, Ministério da Saúde; 2005.

11. Bittencourt RJ, Hortale VA. A qualidade nos serviços de emergência de hospitais públicos e algumas considerações sobre a conjuntura recente no município do Rio de Janeiro. Ciênc Saúde Coletiva 2007; 12:929-34.
12. Sicotte C, Champagne F, Contandriopoulos AP, Béland F, Denis JL, Bilodeau H, et al. A conceptual framework for the analysis of health care organizations performance. Health Serv Manage Res 1998; 11:24-48.

13. Pai M, McCulloch M, Colford J. Diagnostic system atic reviews: a road map (version 3). http://www. medepi.net/meta/guidelines/Diagnostic_System atic_Reviews_Road_Map_V3.pdf (acessado em 12/ Fev/2007).

14. Weiss SJ, Derlet R, Arndahl J, Ernst AA, Richards J, Fernández-Frackelton $\mathrm{M}$, et al. Estimating the degree of emergency department overcrowding in Academic Medical Center: results of the National ED Overcrowding Study (NEDOCS). Acad Emerg Med 2004; 11:38-50.

15. Asplin BR, Magid DJ, Rhodes KV, Solberg LI, Lurie N, Camargo Jr. CA. A conceptual model of emergency department crowding. Ann Emerg Med 2003; 42:173-80.

16. Wilson MJ, Nguyen K. Bursting at the seams: improving patient flow to help America's emergency department. http://www.rwjf.org/research/resear chdetail.jsp?id=1427\&ia=142 (acessado em 23/ Jan/2007).

17. Solberg LI, Asplin BR, Weinick RM, Magid DJ. Emergency department crowding: consensus development of potential measures. Ann Emerg Med 2003; 42:824-34.

18. Donabedian A. Selecting approaches to assessing performance. In: Bashshur R, editor. An introduction to quality assurance in health care. New York: Oxford University Press; 2003. p. 30-8.

19. Shah MN, Fairbanks RJ, Maddow CJ, Lerner EB, Syrett JI, Davis EA, et. al. Description and evaluation of a pilot physician direct emergency medical services diversion control program. Acad Emerg Med 2006; 13:54-60.

20. Patel PB, Derlet RW, Vinson DR, Williams M, Wills J. Ambulance diversion reductions: The Sacramento solution. Am J Emerg Med 2006; 24:206-13. 
21. Schull MJ, Szalai JP, Schwartz B, Redelmeier DA. Emergency department overcrowding following systematic hospital restructuring: trends at twenty hospitals over ten years. Acad Emerg Med 2001; 8:1037-43.

22. Han JH, Zhou C, France DJ, Zhong S, Jones I, Storrow $\mathrm{AB}$, et al. The effect of emergency department expansion on emergency department overcrowding. Acad Emerg Med 2007; 14:338-43.

23. Patovi SN, Nelson BK, Bryan ED, Walsh MJ. Faculty triage shortens emergency department length of stay. Acad Emerg Med 2001; 8:990-5.

24. Schneider S, Zwemer F, Doniger A, Dick R, Czapranski T, Davis E. Rochester, New York: a decade of emergency department overcrowding. Acad Emerg Med 2001; 8:1044-50.

25. Nash K, Zachariah B, Nitschmann J, Psencik B. Evaluation of fast track unit of university emergency department. J Emerg Nurs 2007; 33:14-20.

26. Torres V, Brunett P, Fu R, Lowe R, Schmidt T, Hedges J. Leaving without being seen, boarding, bored, or both? Acad Emerg Med 2007; 14 Suppl 1:S54.

27. Kelen GD, Scheulen JJ, Hill PM. Effect of emergency department (ED) manage acute care unit on ED overcrowding and emergency medical service diversion. Acad Emerg Med 2001; 8:1095-100.

28. Gantt LT. A strategy to manage overcrowding: development of an ED holding area. J Emerg Nurs 2004; 30:237-42.

29. Ganapathy S, Zwemer FL. Coping with a crowded ED: an expanded unique pole for midlevel providers. Am J Emerg Med 2003; 21:125-8.

30. Karriem-Norwood VA, Haley L, Click L. Care management unit impact on emergency department overcrowding. Acad Emerg Med 2006; 13 Suppl 1: S29.

31. Chen P, Sixsmith D. Early treatment unit does not improve hospital length of stay for ED boarders. Acad Emerg Med 2007; 14 Suppl 1:S54.

32. Richardson T, Dick R, Schneider S. Evaluation of an Emergency Department Inpatient Observational Unit. Acad Emerg Med 2007; 14 Suppl 1:S198.

33. Mace SE, Graff L, Mikhail M, Ross M. A national survey of observation units in the United States. Am J Emerg Med 2003; 21:529-33.

34. Tahan H. Managing Emergency Department overload. Nurse Leader 2005; 3:40-3.

35. Doxzon G, Howard-Ducsay J. ED overcrowding: successful action plan of Southern California Community Hospital. J Emerg Nurs 2004; 30:325-9.

36. Killen J, Chan T, Castillo E, Jones K, Guss D. Inpatient nurse staffing to care for ED boarders does not relieve emergency department crowding. Acad Emerg Med 2007; 14 Suppl 1:S82.

37. Castillo E, James K, Guss D, Jones K, Marshall J, Chan T. Impact of mandated nurse-patient ratios on emergency flow in 2 California Emergency Department. Acad Emerg Med 2007; 14 Suppl 1:S83.

38. Celia CA, Vose C, Natale G, Miccuci KD. Leadership development for the front line. J Emerg Nurs 2006; 32:10.

39. Presley D, Robinson G. ED overcrowding and inpatient hold: The Contingency Plan. J Emerg Nurs 2004; 30:208.
40. St. Pierre R. Improving patient flow and patient transfers through implementation of ED nursing voicemail report system. J Emerg Nurs 2004; 30:208.

41. Weintraub B, Hashemi T, Kucewicz R. Creating an enhance triage area improves emergency department throughout. J Emerg Nurs 2006; 32:502-5.

42. Singer A, Viccellio P, Niegelber E, Thode Jr. H, Henry $\mathrm{M}$. Introduction of a stat laboratory reduces $\mathrm{ED}$ length of stay. Acad Emerg Med 2007; 14 Suppl 1: S79.

43. Green LV, Soares J, Giglio JF, Green R. Using queueing theory to increase the effectiveness of emergency department provider staffing. Acad Emerg Med 2006; 13:61-8.

44. Innes G, Grafstein E, Stenstrom R, Harris D, Hunte G. Impact of an overcapacity care protocol on emergency department overcrowding. Acad Emerg Med 2007; 14 Suppl 1:S85.

45. Robinson G, Presley D. Admission-DischargeObservation Unit: surviving ED overcrowding. J Emerg Nurs 2004; 30:211.

46. Barlett J, Cameron P, Cisera M. The Victorian emergency department collaboration. Int J Qual Health Care 2002; 14:463-70.

47. Bertosy DA, Kuszajewski ML, Marsh EE. Directroom: one department's approach to improving ED throughput. J Emerg Nurs 2007; 33:26-30.

48. Maniago E, Ardolic B, Peana J. ED patient flow: Utilizing the six sigma approaches to reduce Emergency Department overcrowding. Ann Emerg Med 2005; 46:S8.

49. Dunn R. Reduced access block causes shorter emergency department waiting times: an historical control observational study. Emerg Med 2003; 15:232-8.

50. Dobbins M, Cockerill R, Barnsley J. Factors affecting the utilization of systematic reviews. Int J Technol Assess Health Care 2001; 17:203-14.

51. Victora CG, Habicht J-P, Bryce J. Evidence-based public healthy moving beyond randomized trials. Am J Public Health 2004; 94:400-5.

52. Des Jarlais DC, Lyles C, Crepaz N. Improving the reporting quality of nonrandomized evaluations of behavioral and public health interventions: The TREND statement. Am J Public Health 2004; 94:361-6.

53. Grimshaw J, McAuley LM, Bero LA, Grilli R, Oxman AD, Ramsay C. Systematic reviews of effectiveness of quality improvement strategies and programmers. Qual Saf Health Care 2003; 12:298-303.

54. Foster AJ. An agenda for reducing emergency department crowding. Ann Emerg Med 2005; 45: 479-81.

55. Bastos FI. Revisão, revisão sistemática e ensaio em saúde pública. Cad Saúde Pública 2007; 23:1252-3.

56. Bittencourt RJ. Pacto pela qualidade no SUS: estudo de viabilidade técnica no subsistema de urgência e emergência. Brasília: Secretaria Executiva, Ministério da Saúde; 2006.

57. Asplin BR, Magid DJ. If you want to fix crowding, start by fix your hospital. Ann Emerg Med 2007; 49:273-4. 
58. Agência Nacional de Vigilância Sanitária. Programa Nacional de Avaliação de Serviços de Saúde. Brasília: Agência Nacional de Vigilância Sanitária; 2007.

59. Lemiuex-Charles L, Champagne F. Introduction: towards a broader understanding of the use of knowledge and evidence in health care. In: Lemiuex-Charles L, Champagne F, editors. Using knowledge and evidence in health care: multidisciplinary perspectives. Toronto: University of Toronto Press; 2004. p. 3-17.

60. Richardson SK, Ardagh M, Gee P. Emergency department overcrowding: the emergency department cardiac analogy model (EDCAM). Accid Emerg Nurs 2005; 13:18-23.

61. Hackenschmidt A. Should access to Emergency Department be limited for "frequent fliers"? J Emerg Nurs 2003; 29:486-8.
62. Frank IC. ED crowding and diversion: strategies and concern from across the United States. J Emerg Nurs 2001; 27:559-65.

63. Haugh R. A true picture of what ails your emergency department. Hosp Health Netw 2004; 78:67-70.

64. Haugh R. Rethinking our pressure-packed emergency departments. Hosp Health Netw 2003; 77:67-70.

65. Sinreich D, Marmor Y. Ways to reduce patient turnaround time and improve service quality in emergency department. J Health Organ Manag 2005; 19:88-105.

Recebido em 12/Ago/2008

Versão final reapresentada em 14/Jan/2009

Aprovado em 22/Jan/2009 